\title{
Der subtile Unterschied zwischen «kompliziert» und «komplex»
}

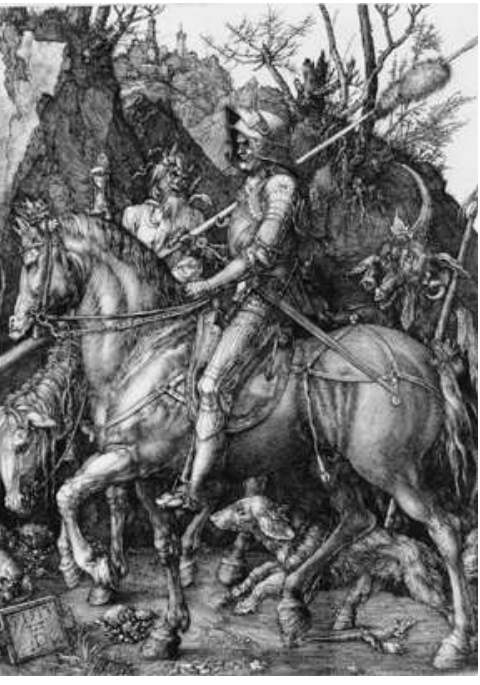

Der Verlust der Komplexität eines Systems kann zu Chaos, aber auch zur Erstarrung führen. Albrecht Dürer, 1513: Ritter, Tod und Teufel (Albertina, Wien).

\footnotetext{
* Prof. Dr. med. Hans Stalder, Facharzt FMH für Innere Medizin FMH, Redaktionsmitglied, ist ehemaliger Direktor der Policlinique de Médecine und des Départements de Médecine communautaire des Hôpitaux Universitaires de Genève.
}

hans.stalder[at]saez.ch
Im September 2001 lenkten mehrere Artikel im British Medical Journal die Aufmerksamkeit auf das Phänomen der Komplexität in Medizin und Gesundheit. Später wurde das Thema der komplexen Systeme in der Allgemeinmedizin im Buch «Complexity in Primary Care» von Kieran Sweeny meisterhaft behandelt [1]. Komplexität stand überdies im Mittelpunkt der Wonca-Konferenz in Basel im Jahr 2009 - und war drei Jahre zuvor das Thema der Abschiedsvorlesung des Autors dieses Artikels [2].

Ein komplexes System zeichnet sich durch Selbstregulierung und das Vermögen zur Anpassung an andere Systeme aus. Man kann es nicht in seine Komponenten zerlegen und neu gestalten, ohne seine Struktur zu zerstören. Ein komplexes System ist durch seine Vergangenheit geprägt und verändert sich im Laufe der Zeit. Geringfügige Eingriffe können unerwartete und starke Reaktionen auslösen. Und schliesslich kann der Verlust der Komplexität eines Systems entweder zu Chaos oder zur Erstarrung führen.

Wenn wir anerkennen, dass physiologische Systeme und folglich unsere Patienten komplexe Systeme sind, hat dies weitreichende Konsequenzen: $\mathrm{Zu}$ nächst bedeutet es, dass der auf die Phänomene der Krankheit fokussierte lineare, kausale Ansatz - der zum Verständnis der Physiologie wichtig ist - nicht zwangsläufig zu ihrer Heilung ausreicht. Die Krankheit muss als Verlust und nicht, wie wir meist annehmen, als Zunahme an Komplexität betrachtet werden. Der Sinusrhythmus als perfekter Ausdruck von Komplexität kann im Krankheitsfall zum Herzflimmern ausarten (Chaos) oder zum Stillstand kommen (Erstarrung und Tod). Folglich befindet sich der gesunde Patient (das gesunde Herz) in einem Zustand maximaler oder optimaler Komplexität. Wenn wir in das komplexe System eines Patienten eingreifen, müssen wir dessen Sensitivität gegenüber früheren $\mathrm{Zu}$ ständen berücksichtigen, als ob es sein eigenes Gedächtnis hätte. Einerseits ist eine restitutio ad integrum im engen Sinne nicht möglich, andererseits reagiert jeder Patient aufgrund seiner früheren Erfahrungen anders, denn der komplexe Organismus passt seine Struktur immer wieder an. Und schliesslich kann man sich auch den Kontakt zwischen Patient und Arzt als ein Aufeinandertreffen zweier komplexer Systeme mit ihrer jeweiligen Vergangenheit vorstellen: Jedes Mal entsteht eine neue Situation, die sich mit der Zeit verändert. Und - wer hätte diese Erfahrung nicht gemacht? - unsere Eingriffe, seien sie auch noch so klein, können unerwartete Folgen haben.

Trotz umfangreicher Literatur über komplexe Systeme haben viele Autoren ihre Bedeutung nicht im- mer verstanden. Wie in der Gemeinsprache wird oft nicht zwischen den Begriffen komplex und kompliziert unterschieden. So haben verschiedene Autoren versucht, die Komplexität der Patienten anhand der Anzahl ihrer Komorbiditäten zu definieren [3, 4]. Andere haben sich bemüht, sozioökonomische Faktoren mitzuberücksichtigen, um den komplexen vom nicht komplexen Patienten abzugrenzen [5]. Dieser Ansatz ist ein typisches Beispiel für das lineare Denken in der Medizin. Man zerlegt ein System in Einzelteile und vergisst dabei, dass das Zusammenfügen der Teile nicht mehr das Ganze ergibt. Paradoxerweise haben die aufgrund ihrer Polymorbidität als komplex betrachteten betagten Patienten just ihre Komplexität verloren: Sie können sich nicht mehr gut an innere oder äussere Einflüsse anpassen und reagieren somit viel empfindlicher auf unsere Eingriffe. Ein Diuretikum, das bei einem jungen Menschen allenfalls eine erhöhte Diurese auslöst, kann bei einem älteren Patienten mit Komorbiditäten zu Hyponatriämie, Hirnödem, Hypotonie, Sturz, Fraktur, Lungenembolie und zum Tode führen. Diese Patienten sind nicht komplexer, sondern durch den Verlust ihrer Komplexität sind sie komplizierter geworden!

Erinnern wir uns: Das Wort Komplikation ist vom lateinischen «complicatio» abgeleitet, was Zusammenwickeln, Vervielfältigung bedeutet, während «complexus» Umschliessung, Umarmung heisst. Eine komplizierte Sache setzt sich daher aus vielen Elementen zusammen, die mithilfe einer linearen Analyse, wie sie häufig der Spezialist vornimmt, separiert werden können. Ein komplexes System vereint hingegen verschiedene Elemente und erfordert einen holistischen Ansatz, wie ihn der Allgemeinmediziner praktiziert. Wir sollten uns um den feinen Unterschied bemühen [6]!

Hans Stalder*

\section{Literatur}

1 Sweeny K. Complexity in primary care. Oxford: Radcliffe Publishing; 2006.

2 Stalder H. Médecine générale - pensée linéaire et complexité. Rev Med Suisse 2006;2:2150-3.

3 De Jonge P, Huyse FJ, Stiefel FC. Case and care complexity in the medically ill. Med Clin North Am. 2006;90:679-92.

4 Weiss KB. Managing complexity in chronic care: an overview of the VA state-of-the-art (SOTA) conference. J Gen Intern Med. 2007;22 Suppl 3:374-8.

5 Grant RW, Ashburner JM, Hong CS, Chang Y, Barry MJ, Atlas SJ. Defining patient complexity from the primary care physician's perspective: a cohort study. Ann Intern Med. 2011;155:797-804.

6 Waldvogel FA, Perone N, Schussele-Filliettaz S, et al. Les malades complexes: de la théorie des systèmes complexes à une prise en charge holistique et intégrée. Rev Med Suisse. 2012;8:1022-4. 